\title{
The Prevalence and Significance of Overt Disseminated Intravascular Coagulation in Patients with Septic Shock in the Emergency Department According to the Third International Consensus Definition
}

\author{
Byuk Sung Ko, M.D. ${ }^{1}$, Hyun Young Cho, M.D. ${ }^{2}$, Seung Mok Ryoo, M.D. ${ }^{3}$, Myung Chun Kim, M.D, Ph.D. ${ }^{1}$, \\ Woong Jung, M.D., Ph.D. ${ }^{1}$, Sung Hyuk Park, M.D, Ph.D. ${ }^{1}$, Chang Min Lee, M.D., Ph.D. ${ }^{1}$, \\ and Won Young Kim, M.D., Ph.D. ${ }^{3}$
}

${ }^{1}$ Department of Emergency Medicine, College of Medicine, Kyung Hee University, Kyung Hee University Hospital at Gangdong, Seoul; '2Department of Emergency Medicine, College of Medicine, Dankook University, Cheonan; ${ }^{3}$ Department of Emergency Medicine, Asan Medical Center, University of Ulsan College of Medicine, Seoul, Korea

Background: The prevalence and prognostic value of overt disseminated intravascular coagulation (DIC) in patients with septic shock presenting to emergency departments (EDs) is poorly understood, particularly following the release of a new definition of septic shock. The purpose of this study was to investigate the prevalence and prognostic value of DIC in septic shock.

Methods: We performed retrospective review of 391 consecutive patients with septic shock admitting to the ED of tertiary care, university-affiliated hospital during a 16-month. Septic shock was defined as fluid-unresponsive hypotension requiring vasopressor to maintain a mean arterial pressure of $65 \mathrm{mmHg}$ or greater, and serum lactate level $\geq 2 \mathrm{mmol} / \mathrm{L}$. Overt DIC was defined as an International Society on Thrombosis and Hemostasis (ISTH) score $\geq 5$ points. The primary endpoint was 28-day mortality.

Results: Of 391 patients with septic shock, 290 were included in the present study. The mean age was 65.6 years, the 28 -day mortality rate was $26.9 \%$, and the prevalence of overt DIC was $17.6 \%(n=51)$ according to the ISTH score. The median DIC score was higher in non-survivors than in survivors (5.0 vs. 2.0, $\mathrm{p}=0.001$ ). Significant higher risk of mortality was observed in overt DIC patients compared to those without $(28.2 \%$ vs. $13.7 \%, p=0.005)$. Multivariable logistic regression analysis identified DIC to be independently associated with 28-day mortality (odds ratio, 2.689 [95\% confidence interval, 1.390-5.201]).

Conclusions: Using the ISTH criteria of DIC, overt DIC in septic shock was found to be common among patients admitting to the ED and to be associated with higher mortality when it is accompanied with septic shock. Efforts are required to identify presence of overt DIC during the initial treatment of septic shock in patients presenting the the ED.

Key Words: disseminated intravascular coagulation; prevalence; shock, septic.

\section{Introduction}

Sepsis and septic shock are grave consequences of infection. Despite the significant improvement in intensive manage-

Received on April 27, 2016 Revised on May 24, 2016

Accepted on August 24, 2016

Correspondence to: Won Young Kim, Department of Emergency Medicine, Asan Medical Center, University of Ulsan College of Medicine, 88 Olympic-ro 43-gil,

Songpa-gu, Seoul 05505, Korea

Tel: +82-2-3010-3350, Fax: +82-2-3010-3360

E-mail: wonpia73@naver.com

*The authors disclose no conflicts of interest.

*The authors declare they have no funding source. ment, sepsis still has shown high morbidity and mortality. [1-4] Definition of sepsis was recently published as lifethreatening organ dysfunction with infection. And organ dysfunction can be identified by an acute increase of $\geq 2$ points in the sequential organ failure assessment (SOFA) score.[5] The new criteria for septic shock include fluidunresponsive hypotension requiring vasopressors to main- 
tain mean arterial pressure of $\geq 65 \mathrm{mmHg}$ with serum lactate level $\geq 2 \mathrm{mmol} / \mathrm{L}$. Disseminated intravascular coagulation (DIC), which leads to fibrin thrombosis, microvascular obstruction, and decreased oxygen delivery resulting in organ failure.[6,7] Although previous studies have reported an association between DIC and mortality of sepsis patients in intensive care units, few studies have evaluated patients with septic shock attending emergency department (ED).[8,9] Furthermore, there is no study about the prevalence and prognostic value of DIC in septic shock patients following the new definition of septic shock.

Therefore, the purpose of present study was to evaluate the prevalence of DIC according to the definition of the International Society on Thrombosis and Hemostasis (ISTH) and determine the predictive value of overt DIC in septic shock patients.

\section{Materials and Methods}

\section{1) Study design}

The present retrospective cohort study was conducted in the academic ED of a tertiary care, university-affiliated hospital in Seoul, Korea that cares for approximately 110,000 patients per year. Intensive care physicians are available $24 \mathrm{~h}$ a day, 7 days a week, for patients who require treatment for sepsis. Because of the retrospective nature of the study, our institutional review board approved the review of patient data before its commencement and waived the requirement for informed consent.

\section{2) Data collection and patient management}

The electronic medical records of all consecutive adult (age, $>18$ years) patients with septic shock admitted to the ED of our hospital between November 2014 and March 2016 were examined. Sepsis was defined as life-threatening organ dysfunction caused by a dysregulated host response to infection.[5] Organ dysfunction was defined as an acute change in the total SOFA score by $\geq 2$ points due to infection. $[5,10]$ Septic shock was defined as a clinical evidence of sepsis with persistent hypotension requiring vasopressor to maintain a mean arterial pressure of $\geq 65$ $\mathrm{mmHg}$ and serum lactate level $>2 \mathrm{mmol} / \mathrm{L}(>18 \mathrm{mg} / \mathrm{dL})$ despite adequate volume resuscitation.[5] The exclusion criteria utilized were as follows: known coagulation disorder, use of anticoagulation medications, do-not-resuscitate order, absence of outcome data, and lack of blood coagulation testing while in ED. Patients with recognized septic shock after $6 \mathrm{~h}$ since ED admission were also excluded. The primary outcome of the present study was 28-day mortality.

Clinical and demographic characteristics of all patients, including age, sex, comorbidities, initial vital signs, laboratory findings, site of infection, and clinical outcomes, were retrieved from electronic hospital records. We used initial laboratory findings, including coagulation testing, from ED. Platelet count, prothrombin time, fibrinogen, and fibrinogen degradation products (FDP) were used to calculate ISTH scores (Table 1). No increase, moderate

Table 1. Scoring system for overt DIC proposed by ISTH

\begin{tabular}{|c|c|}
\hline Score & \\
\hline Platelet count $\left(\times 10^{3} / \mu \mathrm{L}\right.$ & \\
\hline$<50$ & 2 \\
\hline$\geq 50$ and $<100$ & 1 \\
\hline$\geq 100$ & 0 \\
\hline Fibrin-related markers & \\
\hline Strong increase & 3 \\
\hline Moderate increase & 2 \\
\hline No increase & 0 \\
\hline Prothrombin time (s) & \\
\hline$\geq 6$ & 2 \\
\hline $3-6$ & 1 \\
\hline$<3$ & 0 \\
\hline Fibrinogen level (g/mL) & \\
\hline$<100$ & 1 \\
\hline$\geq 100$ & 0 \\
\hline Calculate score & \\
\hline If $\geq 5$, compatible w & \\
\hline $\begin{array}{l}\text { If }<5 \text {, suggestive (no } \\
\text { repeat next } 1 \text { to } 2\end{array}$ & \\
\hline
\end{tabular}


increase, and strong increase in fibrin-related markers were defined as $\mathrm{FDP}<10,10 \leq \mathrm{FDP}<25$, and $\mathrm{FDP} \geq$ $25 \mathrm{mg} / \mathrm{L}$, respectively.[11] DIC was defined as an ISTH score of $\geq 5$. Blood sampling was conducted within 10 min of presentation to ED. Septic shock treatment was administered according to the recommendations of the international guidelines of Surviving Sepsis Campaign. [12] Rapid administration of sufficient amounts of fluids and antibiotics was performed as soon as possible. Other therapies, such as vasopressors and glucocorticoids, were administered as required. Transfusions of fresh frozen plasma or platelets were not performed for the treatment of DIC unless there was an evidence of significant bleeding. Decisions to perform continuous renal replacement therapy, mechanical ventilation, or other interventions were at the discretion of the attending intensive care physician.

\section{3) Statistical analysis}

All data are presented as the mean \pm standard deviation or median with the interquartile range for continuous variables, and as absolute or relative frequencies for categorical variables. Patients who survived to day 28 were compared with patients who did not. Student's $t$ test and Mann-Whitney $U$ test were used to compare continu- ous variables. The chi-square test was used to compare categorical variables. The results of logistic regression analysis for 28-day mortality adjusted for significant factors identified by univariate analysis $(\mathrm{p}<0.2)$ are presented as odds ratios (ORs) and $95 \%$ confidence intervals (CIs). P-values of $\leq 0.05$ were considered statistically significant. All statistical analyses were performed using SPSS for Windows version 18.0 (SPSS Inc., Chicago, IL, USA).

\section{Results}

During the study period, 391 septic shock patients meeting the new definition of septic shock presented to our ED. Of these, we excluded 56 patients with previously known coagulopathy, 9 with do-not-resuscitate orders, 21 whose initial DIC blood tests were not obtained, 11 currently receiving anticoagulation medications, and 4 in whom 28-day mortality data were unavailable. Accordingly, a number of finally included patients with septic shock was 290 (Fig. 1). The mean age of the patients included in the study was $65.6 \pm 12.7$ years, $60.3 \%$ of which were male. Of the 290 patients, 78 (26.9\%) did not survive to day 28 after admission to the ED. Base-

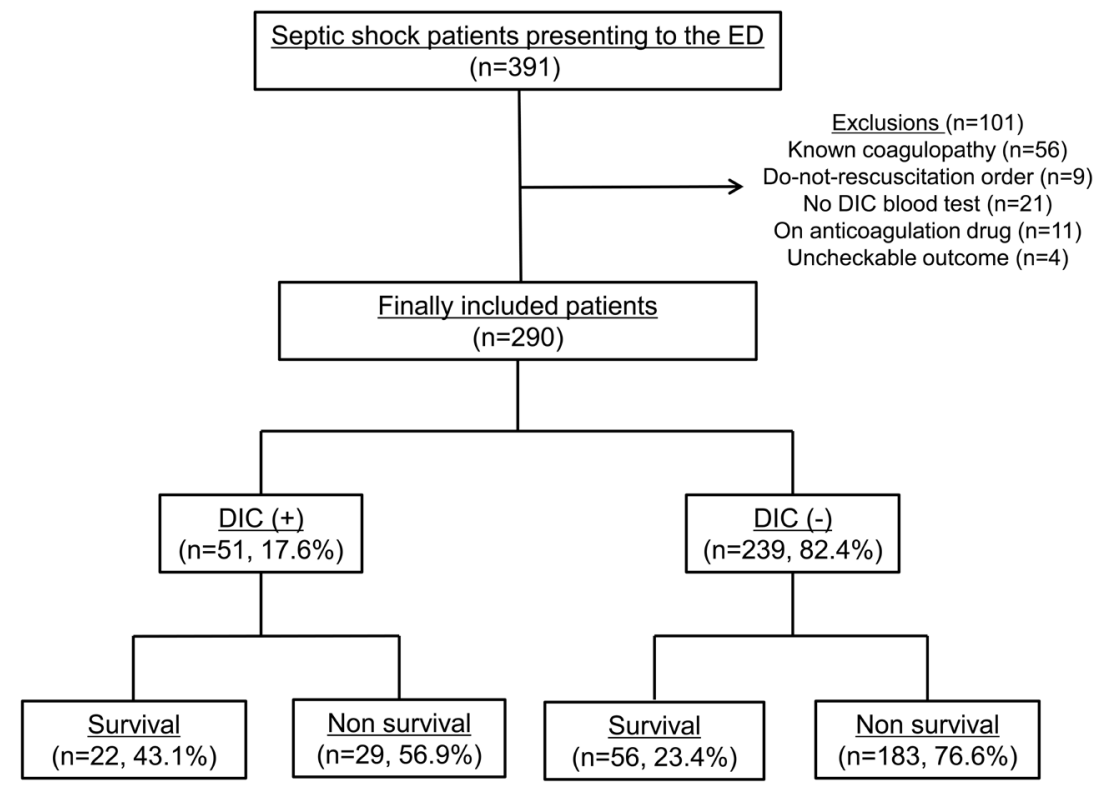

Fig. 1. Patient flow diagram. ED: emergency department; DIC: disseminated intravascular coagulation. 
Table 2. Baseline characteristics and vital signs of septic shock patients according to presence of DIC

\begin{tabular}{|c|c|c|c|}
\hline Variables & No DIC $(n=239)$ & DIC $(n=51)$ & $\mathrm{p}$-value \\
\hline \multicolumn{4}{|l|}{ Demographic factor } \\
\hline Age (years) & $66.0 \pm 12.9$ & $63.4 \pm 11.4$ & 0.173 \\
\hline Male sex & $145(60.7)$ & $30(58.8)$ & 0.875 \\
\hline \multicolumn{4}{|l|}{ Comorbidity } \\
\hline Hypertension & $75(31.4)$ & $14(27.5)$ & 0.620 \\
\hline Diabetes mellitus & $62(25.9)$ & $8(15.7)$ & 0.150 \\
\hline Cardiac disease & $37(15.5)$ & $4(7.8)$ & 0.188 \\
\hline Liver disease & $14(8.4)$ & $7(20.0)$ & 0.062 \\
\hline Neoplasm & $105(43.9)$ & $27(52.9)$ & 0.279 \\
\hline CKD & $12(5.0)$ & $1(2.0)$ & 0.477 \\
\hline COPD & $18(7.5)$ & $0(0.0)$ & 0.050 \\
\hline Immunosuppressed & $120(50.2)$ & $29(56.9)$ & 0.442 \\
\hline \multicolumn{4}{|l|}{ Sites of infection } \\
\hline Lung & $65(27.2)$ & $2(3.9)$ & 0.000 \\
\hline Urinary tract & $34(14.2)$ & $2(3.9)$ & 0.058 \\
\hline Intraabdominal & $41(17.2)$ & $11(21.6)$ & 0.546 \\
\hline Soft tissue & $4(1.7)$ & $2(3.9)$ & 0.285 \\
\hline CNS & $2(0.8)$ & $0(0.0)$ & 1.000 \\
\hline Others & $93(38.9)$ & $34(66.7)$ & 0.000 \\
\hline \multicolumn{4}{|l|}{ Initial vital signs } \\
\hline Systolic blood pressure (mmHg) & $100.6 \pm 30.3$ & $89.0 \pm 20.4$ & 0.001 \\
\hline Diastolic blood pressure (mmHg) & $63.9 \pm 20.8$ & $57.3 \pm 16.0$ & 0.014 \\
\hline Heart rate (beats/min) & $109.0 \pm 27.3$ & $105.7 \pm 26.5$ & 0.431 \\
\hline Respiration rate (breaths/min) & $23.4 \pm 6.4$ & $22.0 \pm 4.0$ & 0.056 \\
\hline Body temperature & $37.4 \pm 1.4$ & $36.9 \pm 1.1$ & 0.002 \\
\hline
\end{tabular}

Values are expressed as mean $\pm \mathrm{SD}$, median and interquartile range, or $\mathrm{n}(\%)$.

DIC: disseminated intravascular coagulation; CKD: chronic kidney disease; COPD: chronic obstructive pulmonary disease; CNS: central nervous system.

line characteristics and vital signs of patients with septic shock, according to the presence of DIC, are summarized in Table 2. No significant differences in age or sex were observed according to 28-day survival (Table 3). Further, no significant differences in the presence of comorbidities or infection site were observed between the two groups. No initial vital signs, except body temperature, differed significantly between the two groups.

Demographic characteristics, initial vital signs, and infection site are presented in Table 3, according to survival at day 28. The overall prevalence of DIC according to ISTH criteria was $17.6 \%$. The prevalence of DIC was higher in patients who did not survive to day 28 compared to those who did $(28.2 \%$ vs. $13.7 \%, p=0.005)$. A significant difference in median ISTH score was observed between the 28 day survival and non-survival groups ( 2.0 vs. $5.0, \mathrm{p}=0.001$; Table 3 ).

Cardiac disease, chronic kidney disease, chronic obstructive lung disease, urinary tract infection, body temperature, DIC frequency, and DIC score were identified to be significantly associated with 28-day mortality using univariate analysis. Stepwise logistic regression analysis 
Table 3. Baseline characteristics and vital signs of septic shock patients according to 28-day mortality

\begin{tabular}{|c|c|c|c|}
\hline Variables & Survival $(n=212)$ & Non survival $(n=78)$ & $\mathrm{p}$-value \\
\hline \multicolumn{4}{|l|}{ Demographic factor } \\
\hline Age (years) & $65.4 \pm 11.8$ & $66.1 \pm 15.1$ & 0.720 \\
\hline Male sex & $126(59.4)$ & $49(62.8)$ & 0.685 \\
\hline \multicolumn{4}{|l|}{ Comorbidity } \\
\hline Hypertension & $65(30.7)$ & $24(30.8)$ & 1.000 \\
\hline Diabetes mellitus & $51(24.1)$ & $19(24.4)$ & 1.000 \\
\hline Cardiac disease & $26(12.3)$ & 15 (19.2) & 0.182 \\
\hline Liver disease & $16(10.5)$ & $5(10.2)$ & 1.000 \\
\hline Neoplasm & $99(46.7)$ & $33(42.3)$ & 0.510 \\
\hline CKD & $7(3.3)$ & $6(7.7)$ & 0.119 \\
\hline COPD & $10(4.7)$ & $8(10.3)$ & 0.100 \\
\hline Immunosuppressed & $110(51.9)$ & $39(50.0)$ & 0.792 \\
\hline \multicolumn{4}{|l|}{ Sites of infection } \\
\hline Lung & 45 (21.2) & $22(28.2)$ & 0.271 \\
\hline Urinary tract & $30(14.2)$ & $6(7.7)$ & 0.163 \\
\hline Intraabdominal & $35(16.5)$ & $17(21.8)$ & 0.304 \\
\hline Soft tissue & $4(1.9)$ & $2(2.6)$ & 0.662 \\
\hline CNS & $1(0.5)$ & $1(1.3)$ & 0.466 \\
\hline Others & $97(45.8)$ & $30(38.5)$ & 0.288 \\
\hline \multicolumn{4}{|l|}{ Initial vital signs } \\
\hline Systolic blood pressure (mmHg) & $98.7 \pm 29.1$ & $98.1 \pm 29.3$ & 0.865 \\
\hline Diastolic blood pressure (mmHg) & $62.5 \pm 19.2$ & $63.3 \pm 22.7$ & 0.772 \\
\hline Heart rate (beats/min) & $107.7 \pm 27.5$ & $110.3 \pm 26.2$ & 0.469 \\
\hline Respiration rate (breaths/min) & $23.0 \pm 6.5$ & $23.4 \pm 4.5$ & 0.631 \\
\hline Body temperature & $37.4 \pm 1.3$ & $37.0 \pm 1.3$ & 0.021 \\
\hline DIC score & $2.0(2.0-3.0)$ & $5.0(5.0-5.0)$ & 0.001 \\
\hline DIC frequency & $29(13.7)$ & $22(28.2)$ & 0.005 \\
\hline
\end{tabular}

Values are expressed as mean $\pm \mathrm{SD}$, median and interquartile range, or $n(\%)$.

CKD: chronic kidney disease; COPD: chronic obstructive pulmonary disease; CNS: central nervous system; DIC: disseminated intravascular coagulation.

was conducted to find independent variables able to predict 28-day mortality. DIC scores were not entered into the logistic regression analysis because of multicollinearity with DIC frequency. The presence of DIC was revealed to be independently associated with higher risk of mortality (OR, 2.689; [95\% CI, 1.390-5.201], p = 0.003; Table 4).

The ORs of prothrombin time and fibrinogen were 1.033 and 0.772 , respectively $(p=0.054$ and $p=0.003$, respec- tively). Platelet count and fibrin-related markers were not significantly associated with higher risk of death (Table 5). Increasing ISTH scores was found to be an association with higher risk of death (Fig. 2).

\section{Discussion}

The prevalence of overt DIC by ISTH criteria, was 
Table 4. Multivariate logistic regression analysis for 28-day mortality

\begin{tabular}{lcc}
\hline \multirow{2}{*}{ Variables } & \multicolumn{2}{c}{ Non-survival } \\
\cline { 2 - 3 } & \multicolumn{1}{c}{ p-value } & \multicolumn{1}{c}{ OR $(95 \% \mathrm{Cl})$} \\
\hline DIC & 0.003 & $2.689(1.390-5.201)$ \\
\hline Adjusted for age, sex, cardiac disease, CKD, COPD, UTI origin infection, body \\
temperature, and DIC. \\
DIC: disseminated intravascular coagulation; Cl: confidence interval; OR: odds ratio.
\end{tabular}

Table 5. OR of each components of ISTH score for 28-day mortality

\begin{tabular}{llc}
\hline \multirow{2}{*}{ Variables } & \multicolumn{2}{c}{ Non survival } \\
\cline { 2 - 3 } & p-value & OR $(95 \% \mathrm{Cl})$ \\
\hline Platelet count & 0.805 & $1.000(0.997-1.002)$ \\
Fibrin-related marker & 0.089 & $1.007(0.999-1.015)$ \\
Prothrombin time & 0.054 & $1.033(0.999-1.068)$ \\
\hline Fibrinogen & 0.003 & $0.772(0.650-0.917)$ \\
\hline
\end{tabular}

OR: odds ratio; ISTH: international society of thrombosis and hemostasis; $\mathrm{Cl}$ : confidence interval.

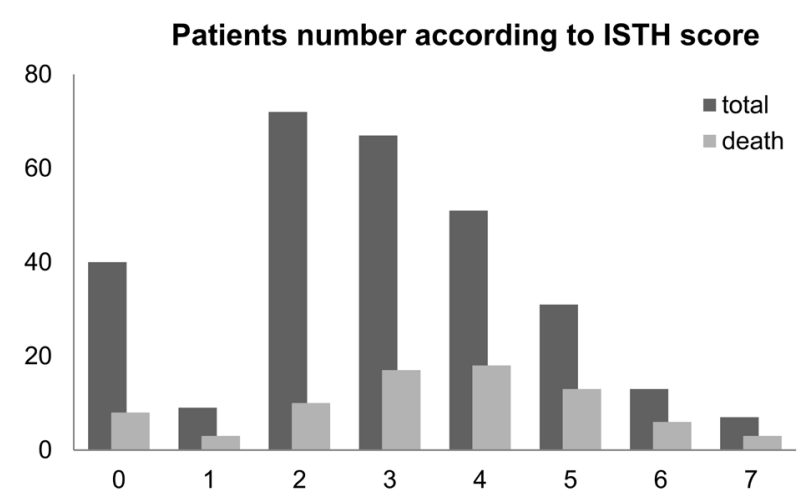

Fig. 2. Patient number according to ISTH score. ISTH: international society of thrombosis and hemostasis.

common among patients attending ED with septic shock diagnosed by new definition (17.6\%). Higher DIC scores were observed in the non-survival group than in survival group at 28 days after ED admission. Furthermore, the presence of DIC in septic shock patients has shown independent association with 28-day mortality.

A new septic shock definition was recently published. Compared with the previous septic shock definition, the new clinical criteria includes a vasopressor requirement and serum lactate $>2 \mathrm{mmol} / \mathrm{L}$ despite volume administration to maintain a mean arterial pressure $\geq 65 \mathrm{mmHg}$.[12]
In that report, risk-adjusted hospital mortality for this group was significantly higher than fluid resistant hypotension requiring vasopressor but with a serum lactate of $\leq 2 \mathrm{mmol} / \mathrm{L}(42.3 \%$ vs. $30.1 \%)$. These results indicate that the new septic shock definition identifies patients with more severe septic shock than the previous definition. Accordingly, the prevalence of DIC and prognostic value of DIC in terms of outcomes should be reevaluated according to the new definition of septic shock. We evaluated the prevalence and prognostic value of DIC using a relatively large sample and strict diagnostic criteria with uniform treatment at a single institution. Our results indicate DIC scores have utility in identifying patients at a high risk of septic shock. Although there has been a controversy regarding the efficacy of treating DIC in patients with sepsis, therapies aimed at DIC may improve outcomes if the presence of DIC is confirmed early.[13-15]

The prevalence of DIC in severe sepsis and septic shock were reported as $18.1 \%$ and $27.5 \%$, respectively, in a previous study by Gando et al.[11] The apparent difference in the prevalence of DIC observed in the present study may be attributable to Gando et al.[11] performing DIC blood tests on days 1 and 4, whereas DIC-related blood sample were drawn on the first day of ED admission in the present study. Although our results indicate a lower prevalence of DIC, the actual prevalence of DIC may be higher if serial evaluations for DIC had been performed. A previous ED-based study reported that overt DIC according to ISTH criteria was found in $13.4 \%$ of patients with sepsis; but, the prevalence overt DIC in septic shock may have been underestimated in this study because all patients of sepsis, severe sepsis, and septic shock patients were included in that study.[6]

The ISTH DIC score is commonly used in clinical setting and associated with adverse outcomes in sepsis patients.[16-20] The result of our study also demonstrate the prognostic value of DIC to identify high risk patients in septic shock. The frequency of patients who had developed DIC in the 28-day mortality group was higher than that of survival group. Further, DIC has shown independent association with 28-day mortality in multivari- 
ate logistic regression analysis, corroborating the results of pre-existing studies. The result of this present study indicate that DIC still has predictive value of identifying high risk patients of sepsis when applied to a new definition of septic shock. Previous studies have reported the areas under the receiver operating characteristic curve of the ISTH criteria in predicting 30-day mortality as 0.819 , which is higher than in the present study (0.629). This difference may be attributable to differences in the study population and the lower mortality despite we only included septic shock patients in our study.[6]

The major limitation of the present study was the single center retrospective study design that reduced the broader generalization of our results. Furthermore, we did not adjust for disease severity during logistic regression analysis and were, therefore, unable to accurately evaluate DIC unable to accurately assume that DIC can predict 28-day mortality. Moreover, differences in individual treatment may have affected the outcomes observed in the present study as the treatment for septic shock was not protocolized, although the management of septic shock was followed by the surviving sepsis campaign. However, the overall 28 -day mortality was $26.9 \%$, which was lower than the $>40 \%$ mortality previously reported when utilizing the previous consensus definition of septic shock.[5] Accordingly, inconsistent treatment did not have a significant effect on patient outcomes. In addition, the proportion of immunosuppressed patient is quite high, so it might be difficult to generalize our result. Lastly, we are unable to exclude the possibility of underestimating the true prevalence of DIC as we used initial DIC laboratory results only. Serial evaluations of the DIC score may increase the accurate evaluation of DIC prevalence and prognostic value observed in our study.

In conclusion, the prevalence of DIC in patients meeting the new definition of septic shock was $17.6 \%$, according to the overt DIC criteria of ISTH. DIC independently associated with 28-day mortality and may have utility in identifying patients at a high risk of death due to septic shock. Efforts are required to identify presence of overt DIC during the early treatment of septic shock in patients attending ED.

\section{References}

1) Dombrovskiy VY, Martin AA, Sunderram J, Paz HL: Rapid increase in hospitalization and mortality rates for severe sepsis in the United States: a trend analysis from 1993 to 2003. Crit Care Med 2007; 35: 1244-50.

2) Kim WY, Huh JW, Lim CM, Koh Y, Hong SB: Analysis of progression in risk, injury, failure, loss, and end-stage renal disease classification on outcome in patients with severe sepsis and septic shock. J Crit Care 2012; 27: 104.e1-7.

3) Ryoo SM, Kim WY, Huh JW, Hong SB, Lim CM, Koh Y, et al: Prognostic value of B-type natriuretic peptide with the sequential organ failure assessment score in septic shock. Am J Med Sci 2015; 349: 28791.

4) Ryoo SM, Kim WY, Sohn CH, Seo DW, Koh JW, Oh BJ, et al: Prognostic value of timing of antibiotic administration in patients with septic shock treated with early quantitative resuscitation. Am J Med Sci 2015; 349: 328-33.

5) Singer M, Deutschman CS, Seymour CW, ShankarHari M, Annane D, Bauer M, et al: The third international consensus definitions for sepsis and septic shock (sepsis-3). JAMA 2016; 315: 801-10.

6) Yin Q, Liu B, Chen Y, Zhao Y, Li C: Prognostic value of the International Society on Thrombosis and Haemostasis scoring system for overt disseminated intravascular coagulation in emergency department sepsis. Infection 2014; 42: 629-37.

7) Levi M: Disseminated intravascular coagulation. Crit Care Med 2007; 35: 2191-5.

8) Dhainaut JF, Shorr AF, Macias WL, Kollef MJ, Levi $\mathrm{M}$, Reinhart K, et al: Dynamic evolution of coagulopathy in the first day of severe sepsis: relationship with mortality and organ failure. Crit Care Med 
2005; 33: 341-8.

9) Voves C, Wuillemin WA, Zeerleder S: International Society on Thrombosis and Haemostasis score for overt disseminated intravascular coagulation predicts organ dysfunction and fatality in sepsis patients. Blood Coagul Fibrinolysis 2006; 17: 445-51.

10) Seymour CW, Liu VX, Iwashyna TJ, Brunkhorst FM, Rea TD, Scherag A, et al: Assessment of clinical criteria for sepsis: for the third international consensus definitions for sepsis and septic shock (sepsis-3). JAMA 2016; 315: 762-74.

11) Gando $S$, Saitoh D, Ogura H, Fujishima S, Mayumi T, Araki T, et al: A multicenter, prospective validation study of the Japanese Association for Acute Medicine disseminated intravascular coagulation scoring system in patients with severe sepsis. Crit Care 2013; 17: R111.

12) Dellinger RP, Levy MM, Rhodes A, Annane D, Gerlach H, Opal SM, et al: Surviving sepsis campaign: international guidelines for management of severe sepsis and septic shock: 2012. Crit Care Med 2013; 41: 580-637.

13) Ogawa Y, Yamakawa K, Ogura H, Kiguchi T, Mohri $\mathrm{T}$, Nakamori Y, et al: Recombinant human soluble thrombomodulin improves mortality and respiratory dysfunction in patients with severe sepsis. J Trauma Acute Care Surg 2012; 72: 1150-7.

14) Kienast J, Juers M, Wiedermann CJ, Hoffmann JN, Ostermann H, Strauss R, et al: Treatment effects of high-dose antithrombin without concomitant heparin in patients with severe sepsis with or without dis- seminated intravascular coagulation. J Thromb Haemost 2006; 4: 90-7.

15) Bernard GR, Vincent JL, Laterre PF, LaRosa SP, Dhainaut JF, Lopez-Rodriguez A, et al: Efficacy and safety of recombinant human activated protein $\mathrm{C}$ for severe sepsis. N Engl J Med 2001; 344: 699-709.

16) Dhainaut JF, Yan SB, Joyce DE, Pettilä V, Basson B, Brandt JT, et al: Treatment effects of drotrecogin alfa (activated) in patients with severe sepsis with or without overt disseminated intravascular coagulation. J Thromb Haemost 2004; 2: 1924-33.

17) Levi M: Another step in improving the diagnosis of disseminated intravascular coagulation in sepsis. Crit Care 2013; 17: 448.

18) Bakhtiari K, Meijers JC, de Jonge E, Levi M: Prospective validation of the International Society of Thrombosis and Haemostasis scoring system for disseminated intravascular coagulation. Crit Care Med 2004; 32: 2416-21.

19) Angstwurm MW, Dempfle CE, Spannagl M: New disseminated intravascular coagulation score: a useful tool to predict mortality in comparison with Acute Physiology and Chronic Health Evaluation II and Logistic Organ Dysfunction scores. Crit Care Med 2006; 34: 314-20; quiz 328.

20) Takemitsu T, Wada H, Hatada T, Ohmori Y, Ishikura $\mathrm{K}$, Takeda T, et al: Prospective evaluation of three different diagnostic criteria for disseminated intravascular coagulation. Thromb Haemost 2011; 105: 40-4. 